\title{
Granulomatous vasculitis of the penis with glomerulonephritis
}

\author{
E. J. Vella \\ B.Sc., M.B.
}

\author{
D. G. WALLER \\ B.Sc., M.R.C.P.
}

\author{
Department of Medicine, University of Southampton, and \\ Department of Histopathology, St Mary's Hospital, Portsmouth
}

\begin{abstract}
Summary
Wegener's granulomatosis is characterized by a necrotizing granulomatous vasculitis of the upper and lower respiratory tract together with glomerulonephritis. Presentation is usually with symptoms referrable to the upper respiratory tract, the glomerulonephritis manifesting itself weeks or months later. A case is reported of a man presenting with a necrotizing granulomatous vasculitis of his penis who subsequently developed glomerulonephritis 18 months later.
\end{abstract}

\section{Case report}

A 67-year-old Caucasian presented with a long history of ischaemic heart disease and was receiving maintenance digoxin and diuretics. In July 1978 he presented with a 4-month history of painless swelling of the glans penis associated with purulent discharge and dysuria. Circumcision and biopsy was undertaken, histology showing marked acute and chronic inflammation with vasculitis and several foci of necrosis. Antibiotic therapy was commenced. ESR was $20 \mathrm{~mm} / \mathrm{hr}$ (Westergren) and renal function normal. The symptoms failed to resolve and partial amputation of the penis was performed in September 1978. Histology showed extensive granulomatous inflammation with vasculitis and areas of necrosis (Figs 1 and 2). In September 1979 he was admitted with a myocardial infarct. Dysuria was again present and, in addition, nocturia, anorexia, nausea and weight loss of recent onset. There were no respiratory symptoms. ESR was $85 \mathrm{~mm} / \mathrm{hr}$ and plasma creatinine $136 \mu \mathrm{mol} / 1$. Disopyramide and spironolactone were added to his medication. While in hospital he developed haematuria, pains in the knees and ankles, and a papular purpuric rash appeared on his buttocks, ankles, feet, elbows and backs of hands. There was erythema over the joints of the hands and elbows. At this stage all drugs were discontinued. The ESR rose to $140 \mathrm{~mm} / \mathrm{hr}$ and renal function steadily deteriorated, the plasma creatinine rising to $549 \mu \mathrm{mol} / \mathrm{l}$. Blood cultures were negative, platelets and clotting tests normal. Fifteen days followingo admission he had a cardiac arrest and died.

Post-mortem examination demonstrated a recent anterior myocardial infarction with thrombotic occlusion of the anterior descending branch of the left coronary artery. There was a cardiac aneurysmo involving the posterior wall of the left ventricle. 옹 The remainder of the left ventricle was severelyo hypertrophied, and the pericardial sac was obliterated by an extensive fibrinous pericarditis. The $\vec{D}$ valves were normal and showed no vegetations. Nog aneurysms of coronary, renal or other arteries were found. The upper respiratory tract was normgl $\infty$ showing no evidence of a necrotizing proces.The lungs were moderately oedematous but c.음 tained no discrete lesions. The bone marrow peared normal. No other significant abnormality⿳亠二口犬 was found elsewhere in the body. Histologically the kidneys showed a focal necrotizing glomerulone- $\frac{\bar{\alpha}}{2}$ phritis (Fig. 3).

\section{Discussion}

A granulomatous vasculitis of either the uppero respiratory tract or lungs is a cardinal feature of both limited and classical Wegener's granulo-3.3. matosis (WG) (Fauci and Wolff, 1973). However, ito has been pointed out that in certain circumstances the diagnosis of WG should be considered in anticio pation of the appearance of respiratory involvement ${ }^{2}$ with a view to starting cytotoxic immunosuppressiveo therapy at an early stage (Baker and Robinson, 1978).

In the case reported here, lesions histologically indistinguishable from those occurring in the respiratory tract in WG were present on the penis while the respiratory tract was uninvolved. Later N the patient was found to have a focal necrotizingo glomerulonephritis which is one of the typical renalo lesions of WG. The delay between initial presentation and subsequent detection of renal involvement is $\stackrel{\infty}{+}$ also reminiscent of WG.

Reed et al. (1963) found focal ulceration of theo skin as an initial feature in $5 \%$ of cases of WG. In 


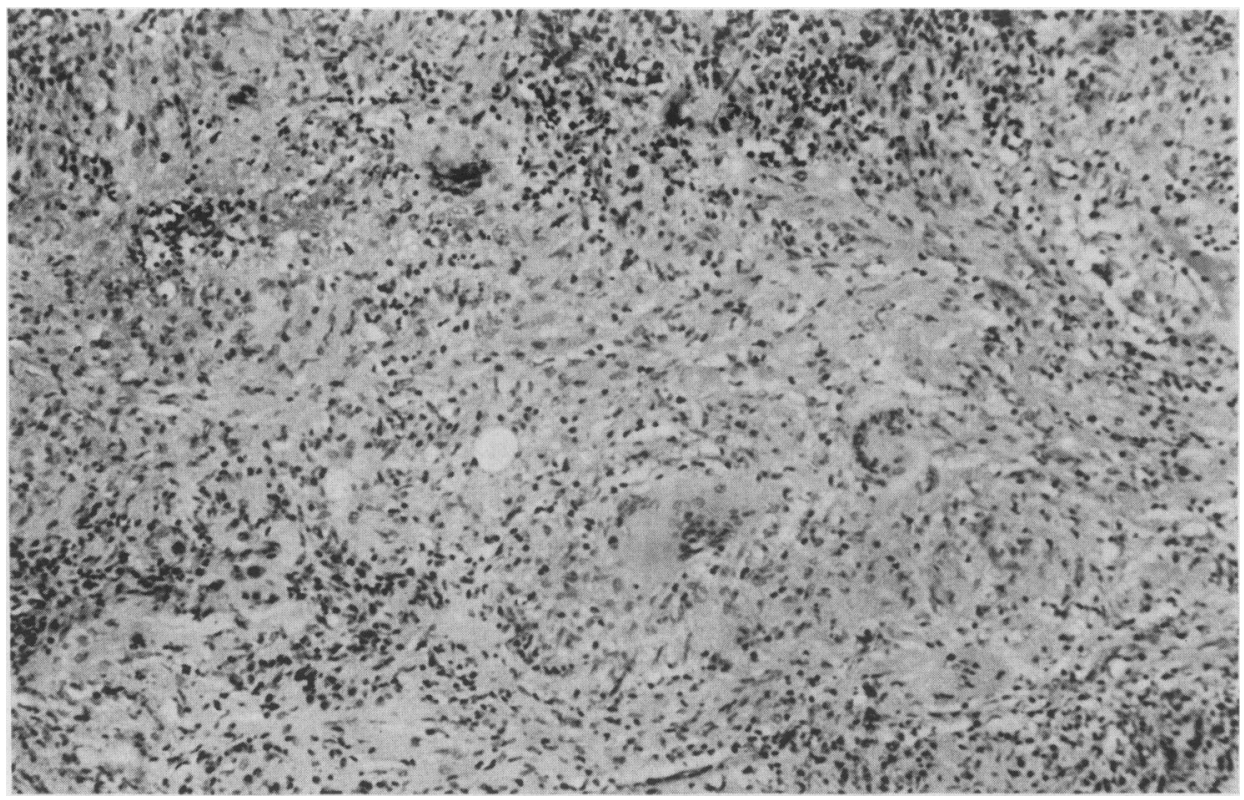

FIG. 1. Histological section from penis showing granulomatous inflammation $(\mathrm{HE}, \times 350)$,

.

о

ญิ

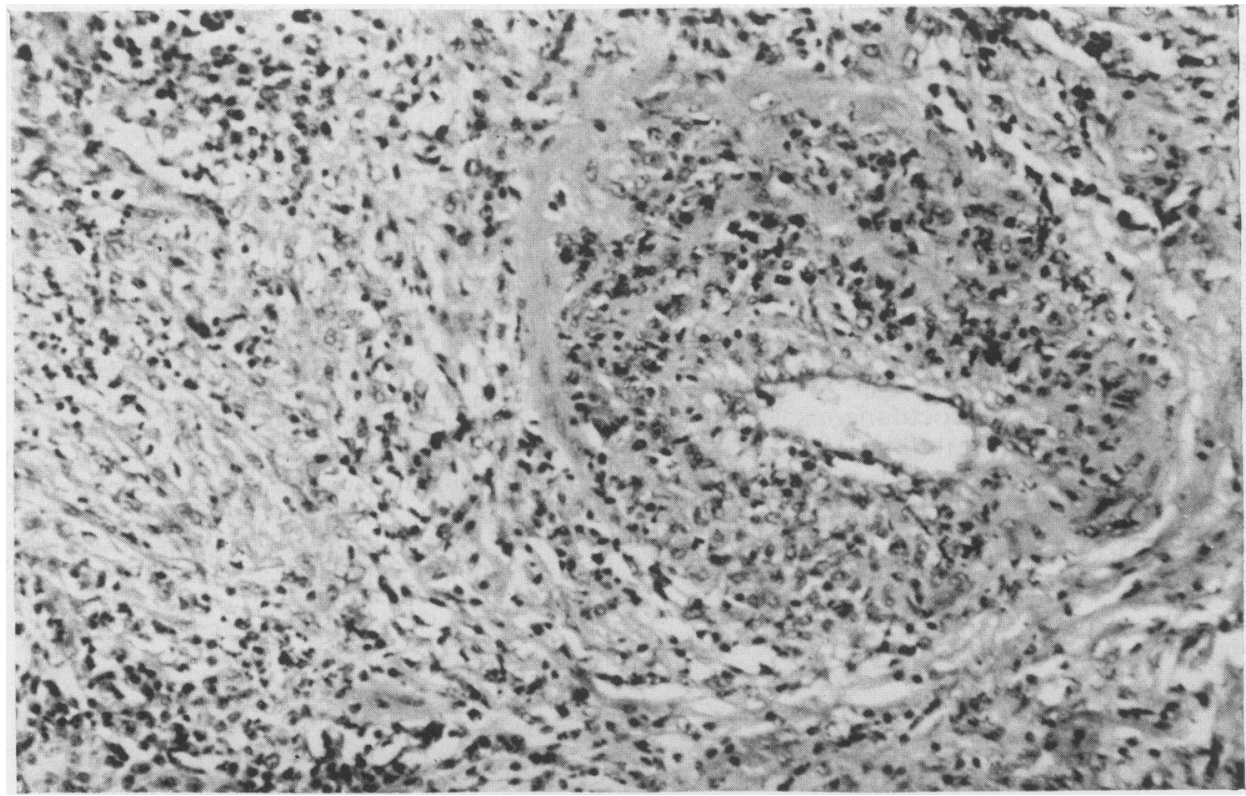

Fig. 2. Histological section from penis showing vasculitis $\left(\mathrm{HE},{ }^{-} \times 350\right)$. 


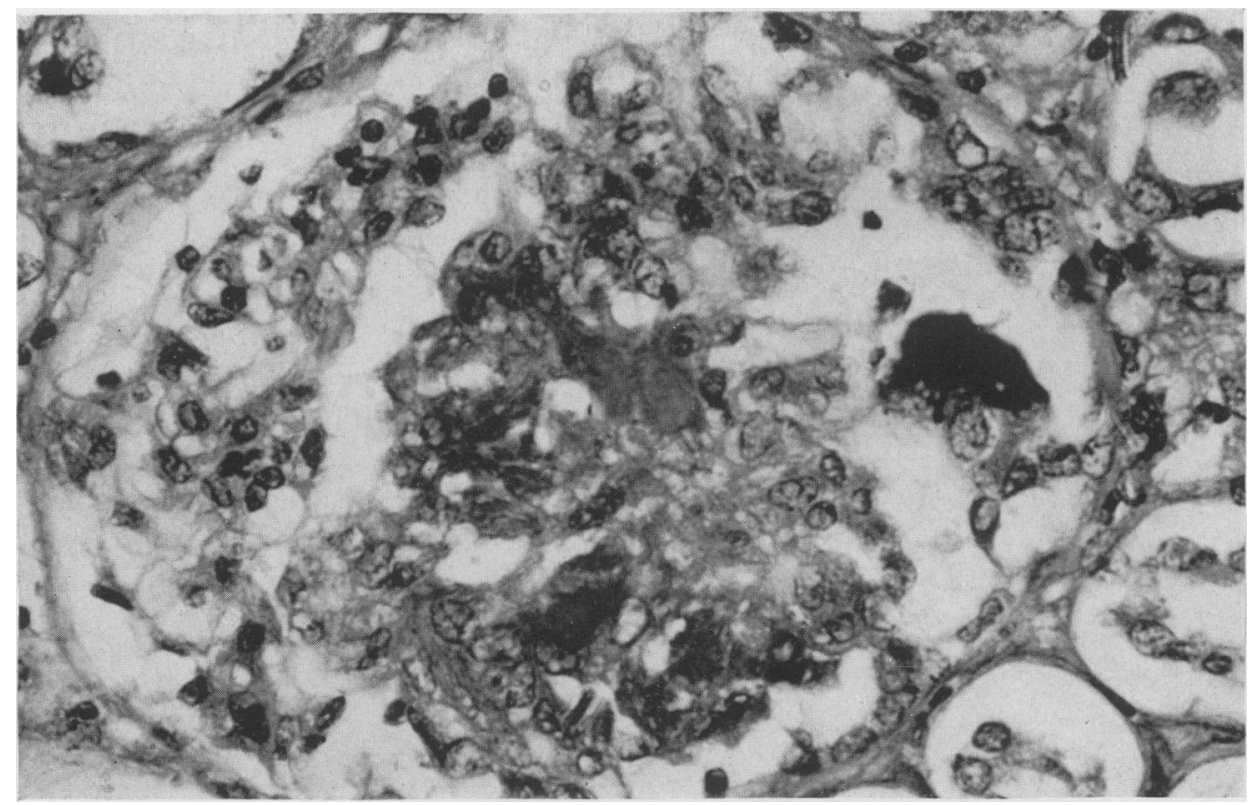

FIG. 3. Histological section from kidney showing focal necrotizing glomerulonephritis (martius, scarlet, blue, $\times 350)$.

the present case, ulceration of the skin of the penis was the first abnormality noted. These authors also described cutaneous lesions in association with terminal renal involvement that closely resemble those present in this patient. Arthralgia or true arthritis of varying severity has also been frequently reported in WG (Fauci and Wolff, 1973). It seems likely therefore that this case represents an unusual presentaton of WG, the initial lesion being situated on the penis instead of the classical site of the upper respiratory tract.

The significance of the penis in this respect may be that it contains a mucocutaneous junction. Lesions of WG in the mouth, another mucocutaneous junction, are well recognized as initial manifestations of the disease (Scott and Finch, 1972). The early occurrence of mucocutaneous lesions in WG has led to the suggestion that if, as seems likely, the disease is caused by a hypersensitivity mechanism, primary sensitization may be occurring at seromucosal surfaces (Edwards and Buckerfield, 1978).
The penis may therefore be an alternative rout sensitization to the mouth.

\section{Acknowledgments}

We thank Professor A. Polak and Mr G. F. Abercromb for their permission to report details of this case, and $\mathrm{Dr} F$ Burston for his help in interpreting the morbid anatomy a histopathology.

\section{References}

BAKER, S.B. \& RoBinson, D.R. (1978) Unusual renal marịfestations of Wegener's granulomatosis. American Journal of Medicine, 64, 883.

EDWARDS, M.B. \& BuCKERFIELD, J.P. (1978) Wegeners granulomatosis: a case with primary mucocutaneogs lesions. Oral Surgery, 46, 53.

FAUCI, A.S. \& WoLfF, S.M. (1973) Wegener's granulomatosis: studies in eighteen patients and a review of the literature. Medicine. Baltimore, $52,535$.

Reed, W.B., Jensen, A.K., Konwaler, B.E. \& Hunter, ㅁ. (1963) The cutaneous manifestations in Wegener's granư: omatosis. Acta dermato-venereologica, 43, 250.

ScOTT, J. \& FINCH, L.D. (1972) Wegener's granulomatoșis presenting as gingivitis. Oral Surgery, 34, 920. 